\title{
2D CHEMODYNAMICAL SIMULATIONS OF LOW-MASS GALAXIES
}

\author{
G. HENSLER AND A. RIESCHICK \\ Institut für Theor. Physik and Astrophysik \\ Universität Kiel, D-24098 Kiel, Germany
}

\begin{abstract}
Because of their low gravitational energy, low-mass galaxies are seriously affected by energetical processes in their interstellar medium, such as supernova explosions, or by gravitational perturbations, e.g., by neighbouring galaxies. This can reasonably explain their variety of morphological types. If the evolutionary timescales of galaxies are predominantly determined by internal processes, the multi-phase character as well as star-gas interactions and phase transitions have to be taken into account. For this purpose we have developed a numerical treatment of the dynamical behaviour of gas and stars, which also accounts for the metal dependence of some processes and which can trace the chemical evolution for different elements. This so-called chemodynamical treatment is described in detail in Theis et al. (1992) and Samland et al. (1997). It considers three stellar components and devides the gas into clouds (CM, with a mass spectrum) and a hot intercloud medium (ICM). Since the element enhancement of the interstellar medium is produced by different processes with different lifetimes of their progenitors, $\mathrm{O}, \mathrm{Fe}$, and $\mathrm{N}$ are used as tracer elements to represent supernovae type II (SNeII), type Ia (SNeIa), and planetary nebulae (PNe) contributions.
\end{abstract} While supernovae form the ICM, PNe only attribute to the CM so that only mixing effects of both gas phases can alter abundance ratios.

Due to limited computer capacities the first chemodynamical simulations of dwarf galaxies could be performed only one-dimensionally so far (see e.g., Hensler et al. 1993, 1998). The recently developed two-dimensional chemodynamical code CoDEx (Samland 1994) was first applied to massive disk galaxies and produced models of which a particular one could represent various chemical and structural observations of the Milky Way with striking agreement (Samland et al. 1997). Here we present the first 2D chemodynamical results for one dwarf galaxy model with $10^{9} M_{\odot}$ and discuss the implications briefly with respect to the importance of abundance mixture processes. The simulations start with a Plummer-Kuzmin density distribution (Satoh 1980). A stationary dark matter halo with a density distribution according to Burkert (1995) is added. The DM central density amounts to $0.062 M_{\odot} p c^{-3}$. The initial spin parameter $\lambda$ is 0.045 .

Besides their morphological differences, dwarf galaxies are characterized by a low metal content and low $\mathrm{N} / \mathrm{O}$ abundance ratios of almost $\log (N / O)=-1.4$ over a large range of $\mathrm{O}$ (Matteucci 1985 ) which is representative for primary $N$ production. Figs. 1-3 show the densities of $N$ and $O$ in the $\mathrm{CM}$ and the ICM, respectively. Because of $\mathrm{N}$ being mixed into the $\mathrm{CM}$ by $\mathrm{PNe}$, while $\mathrm{O}$ is blown into the ICM by SNeII, the density distribution in the CM is different for both elements in the inner parts of the galaxy. While an abundance difference is also expected for the ICM, figs.2 reveal an outflowing ICM structure which is loaded by $\mathrm{O}$ as well as by $\mathrm{N}$. The $\mathrm{N}$ enrichment is due to the evaporation process acting on clouds which are embedded in low-density and high-temperature gas like in the center of the model. Fig. 3 shows this evaporation rate and the $\log (N / O)$ from this simulation of -1.3 is already close to the observed mean value of dwarf galaxies and within the scatter. Condensation of the ICM into clouds closes the material cycle in galaxies, so that the same N/O ratio is appearing in stars as well as in HII regions. Already this first qualitative result provides valuable insight into the importance of self-consistent chemodynamical models which can account for the abundance trends seen in dwarf galaxies. 


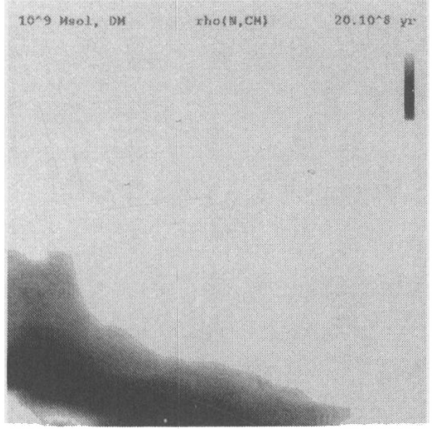

Fig. 1a.

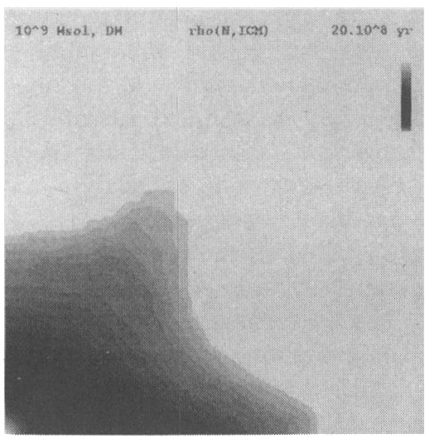

Fig. 2a.

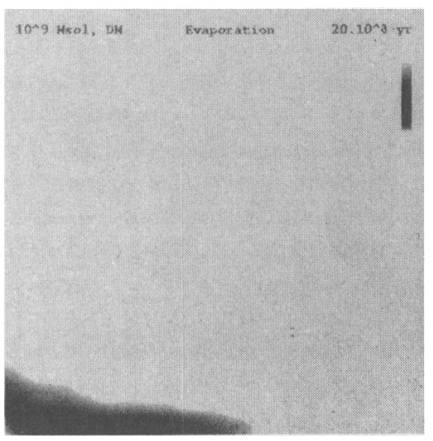

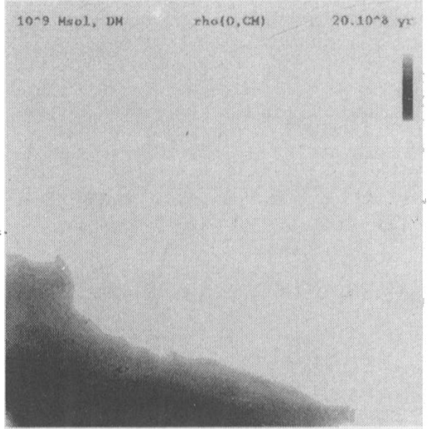

Fig. $1 b$.

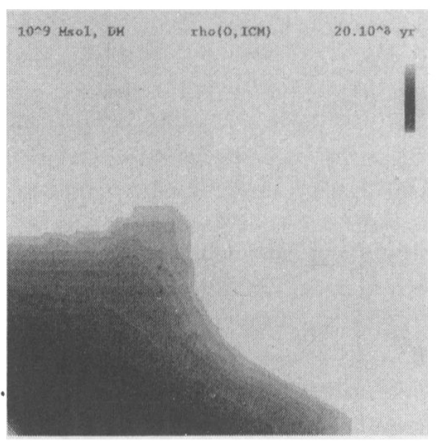

Fig. 2b.

\section{References}

Burkert, A. 1995, ApJ, 447, L25

Hensler, G., Theis, C., Burkert, A. 1993, in Proc. 3d DAEC Meeting, ed. S. Stasińska \& D. Alloin (Paris: l'Obs. de Paris), p. 229

Hensler, G., Theis, C., Gallagher, J.S. 1998, ApJ, in prep.

Matteucci, F., Tosi, M. 1985, MNRAS, 217, 391

Samland, M. 1994, Ph.D. thesis, Univ. of Kiel, Germany Samland, M., Hensler, G., Theis, C. 1997, ApJ, 476, 544

Satoh, C., 1980, PASJ, 32, 41

Theis, C., Burkert, A., Hensler, G. 1992, A\&A, 265, 465

\section{Fig. 3.}

Fig. 1-3. One quadrant of $6.8 \mathrm{kpc} \times 6.8 \mathrm{kpc}$ size between rotation axis (left boundary) and equatorial plane (lower boundary) of a $10^{9} M_{\odot}$ dwarf galaxy with a DM halo (see text) as seen after 2 Gyrs, showing the mass densities of $N$ (figs. a) and $O$ (figs. b) in the CM (figs. 1) and the ICM (figs. 2) as well as the evaporation rate (fig. 3). The grey intensities are chosen arbitrarily reaching the maximum values at the center and the minimum (close to 0 .) in the outermost region (light gray). 